\title{
La marine française au 18 Brumaire
}

Pierre Lévêque

\section{(2) OpenEdition}

Journals

Édition électronique

URL : https://journals.openedition.org/ahrf/289

DOI : 10.4000/ahrf.289

ISSN : $1952-403 X$

Éditeur :

Armand Colin, Société des études robespierristes

Édition imprimée

Date de publication : 1 décembre 1999

Pagination : 639-661

ISSN : 0003-4436

\section{Référence électronique}

Pierre Lévêque, «La marine française au 18 Brumaire ». Annales historiques de la Révolution française [En ligne], 318 | octobre-décembre 1999, mis en ligne le 11 avril 2006, consulté le 22 avril 2022. URL: http://journals.openedition.org/ahrf/289 ; DOI : https://doi.org/10.4000/ahrf.289

Ce document a été généré automatiquement le 22 avril 2022.

Tous droits réservés 


\title{
La marine française au 18 Brumaire
}

\author{
Pierre Lévêque
}

1 Retracer la situation de la marine française en 1799 revient à dresser un sombre tableau. Du golfe de Gênes à Groix, de l'Irlande à Aboukir, on ne peut qu'égrener les défaites. Il convient de préciser cette vision, de retracer l'immense détresse de la marine. La correspondance des commandants d'armes, des chefs de mouvements, des officiers généraux qui commandent des divisions n'est qu'un longue série de plaintes sur la misère de la marine. Cette pénurie entraine de graves difficultés dans une opération qui fut toujours difficile, la levée des matelots. Quand bien même ces matelots parviennent dans les ports, un grand nombre ne pense qu'à déserter. Les sentiments des marins, face à un régime qui paraît les abandonner, nous demeurent très mal connus. Nous ne pouvons que retracer les discours qui sont prononcés au cours de nombreuses cérémonies, discours et pratiques qui manifestent un grand attachement à la République. Cependant la mise en place des nouvelles institutions, après le coup d'État, semble bien accueillie même si les procédures mises en œuvre ne facilitent pas l'expression d'une opposition.

Une marine ruinée

2 Sur le papier la situation de cette marine n'est pas désespérée puisqu'elle peut aligner en 1799, malgré Aboukir, quarante-neuf vaisseaux et cinquante-quatre frégates. Mais il s'agit de bâtiments bloqués dans les ports et pour la plupart incapables de prendre la mer. Le problème que doit affronter cette marine est avant tout un problème financier. En thermidor an $\mathrm{V}$ une note pour le ministre ${ }^{\mathrm{A}}$ en révèle l'extraordinaire détresse. Pour ce mois de thermidor il manque 287000 livres, valeur métallique. Truguet qui commande à Brest a besoin, durant la période comprise entre le 21 brumaire et le 29 messidor, pour les subsistances, la paie des ouvriers, les soldes, de 16300000 livres alors que la Trésorerie nationale ne lui en a versé que 5450000 . Il ne s'agit que des dépenses courantes et « on ne parle point ici de la dette qu'a contractée le département de la marine et des colonies depuis l'émission du papier-monnaie jusqu'au 1er vendémiaire an $\mathrm{V}$ qui s'élève à la somme de 75 millions en numéraires ». Comme ses collègues le ministre de la marine doit recourir à des expédients et demande l'autorisation de délivrer des ordonnances sur la coupe des bois et sur les quatre 
derniers sixièmes des biens nationaux. De plus, la marine considère qu'elle est beaucoup plus mal traitée que l'armée et le ministre s'indigne de cette injustice: « Ce qui l'effraye le plus douloureusement, c'est d'apprendre que dans un même département, dans un même port, un même lieu, la troupe et les agents de l'administration de la guerre soient payés exactement et que la troupe et les agents de la marine ne le soient pas.»

3 Truguet nous fournit un bel exemple de la détresse de la marine confrontée au manque de moyens financiers et à l'incertitude politique. En l'an V, l'amiral envoie une « lettre importante à mettre sous les yeux du Directoire réuni... Je peux vous indiquer, citoyens directeurs le moyen le plus sûr, le plus prompt, le plus efficace de faire cesser toutes vos inquiétudes, c'est d'avoir de l'argent et d'en envoyer... Aujourd'hui encore tout était préparé pour une nouvelle expédition décisive en Irlande, tout était concerté et j'avais trouvé des fonds considérables mais les événements imprévus survenus dans l'ensemble du gouvernement ont tout paralysé » et Truguet conclut : «De l'argent, de l'argent en poste et vous sauvez tout " '. Le même Truguet, lorsqu'il exerce les fonctions ministérielles souligne la différence entre un budget théorique et la réalité des faits, la " différence qui existe entre la quotité nominale de fonds affectés au département de la marine et ceux qu'il reçoit effectivement " '. De plus les moyens de paiement ne sont que des « délégations qui ne donnent lieu qu'à des recouvrements fort retardés, que souvent même les prescriptions sont assignées sur des produits qui n'existent pas. » “

En ventôse an VI, le ministre Pléville le Pelley se rend à Brest et ne peut que constater l'impossibilité de mettre, comme prévu, trente vaisseaux en rade. Le ministre doit avouer qu'on n'a pu effectuer les levées de marins car on n'aurait pu les payer et les nourrir. "C'eût été déterminer la famine dont ce port a été menacé depuis deux mois » s. Les troupes d'artillerie ne sont pas au complet, on manque de chanvre, de cordage, d'approvisionnement, de merrains pour les futailles. Cinq vaisseaux seulement sont en état de prendre la mer alors que le port est bloqué par huit vaisseaux anglais. Le ministre avoue que, même s'il disposait de douze vaisseaux, il ne les ferait pas sortir de crainte que, poussés par des vents d'est, ils ne puissent rentrer à Brest si la croisière anglaise recevait des renforts. Le 1er ventôse an VI, le ministre de la marine écrit au Directoire : «Il y a trente-cinq jours que je n'ai pas eu un sol à ma disposition ” ‘. Le ministre réclame donc, en particulier pour les soldes, du numéraire.

5 L'année suivante la situation ne s'est pas améliorée. En nivôse an VII, le Directoire ordonne l'armement de vingt-quatre vaisseaux à Brest. Morard de Galle, commandant des armes dans ce port et l'ordonnateur Najac manquent de munitions navales et rappellent «la disette absolue... l'épuisement de nos magasins » 'et réclament des fonds. En frimaire le nombre de vaisseaux est ramené à quatorze mais les autorités maritimes se plaignent au ministre. « Notre situation est telle que depuis quatorze mois le service n'est soutenu que par le maintien d'un ordre sévère et par le crédit personnel de l'ordonnateur... Vous nous annoncez des fonds, il est on ne peut plus instant qu'ils arrivent " '. Dans les mois suivants devant le manque de cordage, de chanvre, de caisses pour le biscuit, de merrains pour les futailles, on doit désarmer des vaisseaux pour parvenir à en armer trois. De toute façon, les vaisseaux en partance ne peuvent être calfatés par manque de braye. Dans une lettre personnelle au ministre, l'ordonnateur affirme qu'il souhaite quatorze millions pour les besoins de Brest : Dans la correspondance reviennent sans cesse les plaintes sur la misère de Brest qui « est dans le dénuement le plus extrême... Il est difficile de se faire idée de notre dénuement en 
cordages comme en moyen d'en fabriquer. Il ne peut être comparé qu'à l'épuisement total de nos finances et de toutes nos ressources. ${ }^{10}$

6 Cette situation n'est pas propre à Brest et le commandant des armes à Lorient, l'amiral Nielly attire l'attention du ministre sur « ce port qui est dans la misère la plus affreuse ce qui est d'autant plus dangereux que les Chouans ne sont pas très éloignés " ". Quant au chef des mouvements de Nantes sa situation n'est pas plus enviable, «le commissaire principal me dit chaque jour qu'il n'a pas un sol en poche" ". En vendémiaire an VII, l'ordonnateur du port de Rochefort s'avoue incapable d'armer deux frégates dans un port «absolument dénué des objets les plus importants et nécessaires aux armements projetés " ". Le port d'Anvers est dans une situation aussi pitoyable, l'ordonnateur écrivant au ministre qu'il est « on peut plus mal approvisionné ou pour mieux dire sans approvisionnement. " "

7 Les autres ports semblent moins infortunés même si Bayonne, qui dispose de bois, manque de cordage et ne peut donc mettre à l'eau la frégate Thémis. Le commissaire de marine affirme cependant : "Je suis depuis longtemps sans argent comme sans crédit » ". Bordeaux envoie à Brest tous les fils carrets, grelins et aussières ainsi que les vivres de campagne dont dispose ce port. Les forces navales en Italie sont dans un véritable " état de détresse " ". L'arsenal de Toulon semble le moins dépourvu car l'ordonnateur prétend pouvoir fournir des mâts, des ancres et des cordages à douze vaisseaux de soixante-quatorze et six frégates mais au prix d'expédients: "Je ne suis parvenu à préparer les moyens qui sont aujourd'hui à ma disposition qu'en employant tous les fonds des invalides et des prises, ceux destinés pour l'approvisionnement de Malte et $30000 \mathrm{~F}$ que je suis parvenu à soutirer du payeur de la marine... Il est donc urgent que vous mettiez le plus tôt possible des fonds à ma disposition ». Cette crise ne semble pas propre à la marine comme le montre la pénurie de canons à Nantes, pénurie due au fait qu'Indret ne reçoit plus de fonte du Périgord et que de toutes façons, la pompe à feu est indisponible pour plus de deux mois. Un des rares témoins à présenter un tableau moins sombre est l'amiral Bruix qui vient prendre le commandement de la flotte à Brest: «La caisse de la marine est dans la meilleure situation, le prêt des troupes, la solde des officiers, celles des ouvriers et marins, enfin toutes les dépenses relatives aux individus sont acquittées avec exactitude " ". L'amiral doit rapidement déchanter. Passé en Méditerranée, il compte se refaire à Toulon où, d'après le ministre, « vous trouverez toutes les ressources qui pourront vous être nécessaires ", mais il ne peut que constater que «l'ordonnateur de Toulon est absolument sans fond " ". Il doit lui même avancer $300000 \mathrm{~F}$ sur les fonds qu'il a embarqués à Brest.

8 Les premiers touchés par le manque de fonds sont les ouvriers qui ne perçoivent que très irrégulièrement leurs salaires. À Brest, Morard de Galle et Najac font placarder une affiche le 1er ventôse an VII affirmant que l'arriéré est entièrement à jour, mais à la fin du même mois ils doivent avouer au ministre que le mois de pluviôse et le mois courant ne sont toujours pas payés ". À Rochefort, en vendémiaire an VIII, les ouvriers ne sont pas payés depuis quatre mois. La situation est plus dramatique encore à Lorient où, au début de l'an VIII, les arriérés de salaire remontent à cinq mois, et surtout à Nantes où les ouvriers ne sont plus payés depuis huit à dix mois et refusent de travailler : «Les ouvriers ne veulent plus rien faire faute de moyens de subsistance " ${ }^{20}$. La frégate $L a$ Chiffonne ne peut être réparée car on ne trouve plus d'ouvriers: "Les malheureux ne 
peuvent faire subsister leurs familles, la plupart sont obligés de vendre le peu d'effets qu'ils avaient. »

9 Les ordonnateurs se chargent de nourrir les ouvriers qu'ils ne peuvent payer mais à Rochefort, les boulangers menacent de ne plus fournir de pain à partir du 1er brumaire s'ils ne reçoivent pas de fonds. L'ordonnateur se propose d'avoir recours à un emprunt "que j'ai reculé tant que j'ai pu pour conserver une dernière planche dans le naufrage " ". À Toulon, les autorités maritimes décident de fournir du pain sur les vivres de la marine à des ouvriers qu'elles ne peuvent payer et qui ont « des besoins journaliers pour leur existence" ". La situation est d'autant plus inextricable que, lorsque l'ordonnateur parvient à disposer de fonds, il ne peut les faire accepter par les négociants et munitionnaires de Marseille qui refusent de prendre du papier. La situation est analogue à Brest où « on refuse de recevoir le payement en traites sur Paris depuis que celles que j'ai tirées sur le banquier Féline (?) éprouvent des retards et qu'elles reviennent à protest. " "

10 Les magasins étant vides les marins sont nourris avec des vivres dont la qualité laisse à désirer. "Il est affligeant que le défaut d'approvisionnement de nos magasins nous mette dans l'impérieuse nécessité de faire consommer à nos équipages des vins et du biscuit de journalier d'une très mauvaise qualité ce qui aggrave les privations multipliées de nos marins. " ${ }^{24}$

11 Les soldes des marins ne sont pas plus régulièrement versées à Lorient comme pour les équipages de l'Argonaute et des frégates la Cocarde et la Sémillante à qui dix-huit mois de solde sont dus. À Rochefort «il se trouve des bâtiments armés à qui il est dû aux équipages huit à neuf mois " ${ }^{25}$. Les autorités n'osent faire appareiller ces bâtiments par crainte de la désertion et de mutineries. Il en va de même à Nantes où le chef des mouvements hésite à faire sortir deux goélettes car «il en résulterait de nombreuses défections dans un port où il n'y a pas moyen de s'y opposer $" ~ "$.

12 Mal payés, mal nourris, les marins protestent, en particulier à Brest. "On réclame la solde à grands cris, on ne veut pas de viande salée, on demande du tabac " ". Morard de Galle, alors commandant des armes craint, en messidor an V, une mutinerie. Déjà sur le Formidable les hommes refusent de faire l'exercice.

Une marine désertée

13 Liée au manque d'argent, la pénurie d'hommes est un autre problème. Selon Morard de Galle, l'escadre de Brest manque de 8000 marins et officiers mariniers en nivôse an VII alors que l'ordonnateur de ce même port avance le chiffre de 7000 en ventôse. Un des problèmes fondamentaux de la marine française, le manque de gens de mer, se pose ici avec acuité. Morard de Galle devant la pénurie des quartiers bretons veut se tourner vers le Havre et Cherbourg. Il est vrai que, si l'on en croit le chef des mouvements de Nantes, "mère nourricière de Brest " ${ }^{2 n}$, il ne peut plus compter sur la contribution de ce port qui ne peut plus lui fournir d'hommes étant donné «l'état d'épuisement où se trouve en marins l'arrondissement de Nantes par les armements que nous avons faits et notamment par la quantité de matelots que nous avons envoyés à Brest depuis cinq mois " ". Il en va de même à Bordeaux où les équipages des corvettes en stationnement au Verdon sont réduits aux trois quarts pour être envoyés à Brest avec tous les officiers mariniers et marins disponibles. Ces hommes sont groupés en brigade de cinquante hommes et escortés par des troupes de ligne. En effet, le commissaire de Bordeaux souligne que les «marins du Midi marquent depuis longtemps une répugnance pour les 
provinces du Nord $"{ }^{\circ 0}$. À Rochefort, pour fournir trois cents bons matelots à Brest, on doit désarmer la frégate La Volontaire, les corvettes La Vénus et La Diligente ainsi que huit bateaux canonniers ". En germinal an VII, Bruix qui commande l'escadre, constate qu'il ne peut appareiller de Brest car il lui manque 6000 marins. Il recourt à un moyen de fortune car il propose de " compléter les équipages à l'aide d'un assez grand nombre d'ouvriers du port qui ont navigué " ". L'appel à d'autres quartiers maritimes, les siens étant épuisés, est également la solution préconisée par le commandant des armes de Toulon qui demande en prairial an VII des marins en provenance de Lorient, Rochefort et Brest, marins que ces régions sont bien incapables de lui fournir.

Non seulement les marins ne sont pas suffisamment nombreux mais ceux qui arrivent dans les ports ne sont pas toujours des hommes habitués à la mer : "C'est avec peine que je vois qu'il n'en arrive qu'en très petit nombre et que la plupart de ceux qui sont embarqués en sont à proprement pire que des mauvais marins particulièrement ceux qui sont envoyés de Paris" ". La solution que proposent certains commandants d'unités, solution évidemment impraticable est d'augmenter le nombre d'hommes, pour suppléer à la qualité par la quantité comme le demandent les commandants des frégates la Sémillante et la Corneille à Lorient en floréal an VII. C'est plutôt une solution inverse qui s'impose comme à Brest où Morard se résout à réduire les équipages des vaisseaux de 80 à 750 hommes et ceux des 74 à 600 hommes.

Toutes les autorités sont unanimes, la cause première de ces désertions massives est le retard des paiements. L'ordonnateur de Brest estime, en ventôse an VIII, qu'environ 1100 hommes de mer sont absents et relate au ministre que les désertions sont continuelles, 100 en cinq jours à la fin de ventôse, 208 entre le 10 et le 20 frimaire. À Toulon, où l'escadre est absente, pour armer les bâtiments restant en rade il faudrait 2947 hommes, il en manque $976^{\text {". }}$. Les mesures de rigueur sont inefficaces. À Lorient, "la désertion continue de plus en plus belle à bord de la division du capitaine Villemadrin... Quoique tout le monde soit consigné, les hommes se jettent à la mer et parviennent à s'échapper " ". Ces désertions peuvent être favorisées, si l'on en croit le commissaire du Directoire dans le Calvados, par les ennemis du gouvernement qui, dans ce département, font pression sur les femmes des marins partis à Brest pour que, dans leurs lettres, elles les poussent à déserter ".

En messidor an VII, 152 hommes sont présents sur la frégate la Pensée et 9 sont à l'hôpital pour un effectif théorique de 366. Si l'état-major est au complet, il manque 26 mousses sur 36, 89 matelots sur 160, 64 soldats de garnison sur 89 et 13 chefs de pièce. Dans de telles conditions, il est impensable que ce bâtiment prenne la mer pour affronter l'ennemi.

La situation est aggravée par la concurrence des corsaires comme l'affirme l'ordonnateur de Brest : « Malgré toutes les mesures prises pour empêcher la désertion, elle continue d'avoir lieu et continuera sans doute tant qu'il y aura des corsaires en armement dans le voisinage des ports militaires" ". Si l'on en croit le même ordonnateur, qui propose un embargo sur les corsaires tant que les équipages ne seront pas au complet, il s'agit d'une véritable organisation. Les corsaires « apostent des gens affidés aux passages des grandes routes les plus fréquentées par les gens de mer, ils les arrêtent et les conduisent dans des hôtelleries voisines, là par des promesses séduisantes, par des avances considérables, ils parviennent aisément à s'en rendre maitres, ils s'emparent de leurs ordres de route et de leurs hardes et ils cachent ces hommes en lieu sûr jusqu'au départ... En présence du commissaire des individus de 
toutes professions ramassés au hasard et on leur compte en sa présence une somme quelconque à titre d'acomptes de leurs avances et qui n'est en effet que le prix de la supercherie à laquelle ils se prêtent. Cette revue aussitôt terminée, on expédie le corsaire et aussitôt qu'il est en rade et sous voiles, les matelots embauchés montent à bord d'un côté tandis que les marins supposés en descendent de l'autre " ". À Rochefort, l'amiral Martin estime à 1270 hommes, en grande partie des matelots d'élite, le nombre d'hommes partis s'engager dans les corsaires de Bordeaux. Le commissaire de marine de ce port semble désarmé devant les subterfuges employés. Les corsaires « emploient tous les moyens pour jeter dans l'erreur les commissaires de l'inscription maritime. Tantôt ils se barbouillent le visage, tantôt ils se présentent avec un certificat du bureau central qui constate qu'ils sont manœuvres, ouvriers, laboureurs et ces certificats ont été délivrés à de vrais manœuvres qui les vendent aux marins. ${ }^{30}$

Le mal est général si l'on en croit la mésaventure du capitaine de l'aviso le Bonaparte de Toulon. Cet officier, pour empêcher toute désertion, ne laisse aller à terre que ses huit canotiers mais ceux-ci désertent, l'obligeant à regagner son bord seul avec le patron du canot ". La situation est analogue à Ancône où les marins se jettent à la mer la nuit pour rejoindre les corsaires. Le capitaine de vaisseau Le Mangonnet ne sait comment armer le seul des trois navires vénitiens qui soit en état de prendre la mer. Pour compléter l'équipage de la goélette la Cibèle il a été contraint de prendre celui du chasse-marée Hoche et une partie de celui du brick le Rivoly".

Les troubles intérieurs rendent la situation des ports bretons particulièrement dramatiques. À Lorient, il n'est plus possible de trouver des guetteurs sur les côtes devant l'activité des Chouans. Les ports semblent coupés du reste du pays. En brumaire an VIII, Brest et Lorient ne communiquent plus que par la voie maritime, toutes les routes étant coupées par les Chouans. Le commandant des armes de Brest doit garder le paquet de dépêches qu'il a reçu de Cayenne, "paquet que je vous aurais adressé par un courrier extraordinaire si j'avais été assuré qu'il eût pu passer sans être arrêté par les Chouans et si le manque absolu de fonds de la caisse de la marine avait permis cette dépense" ". C'est à Nantes que la situation est la plus préoccupante. À la fin de brumaire an VIII, le chef des mouvements annonce que la dernière lettre qu'il a reçue de Brest date du 3 du même mois. Les communications avec Paris, Chartres et le Mans sont pratiquement coupées. Les Chouans sont «maîtres de la campagne des départements du Morbiban comme dans ceux de la Loire-Inférieure et du Maine-etLoire " ". Entre Port-Malo et Paris, la correspondance est tout aussi difficile puisque, en brumaire an VIII, le courrier a vingt-cinq jours de retard.

Les officiers ne sont pas mieux lotis que les équipages. À Rochefort, «il est dû quatre mois aux officiers, la plus grande partie ont de la famille et éprouvent les plus grandes privations " ". À Toulon, les retards de paiement sont si importants que "plusieurs officiers ont vendu linge et bijou " ". D'ailleurs depuis quelques mois ce port manque cruellement d'officiers compétents si l'on en croit l'ordonnateur ".

21 Si l'on en croit le corsaire Plucket, alors employé comme officier de marine, «le gouvernement ne s'inquiétait pas davantage de la paie, car moi-même, avec tout mon état-major de la corvette la Jalouse, nous passâmes plus d'une année sans recevoir un seul denier pour nos appointements de solde ou de traitements de table. Les équipages ne furent pas mieux traités" ". La présence d'un corsaire parmi les officiers nous montre que le corps a été profondément bouleversé. Devant une émigration qui devient 
massive à partir du printemps 1792, les gouvernements successifs ont fait appel, pour combler les vides, d'abord à d'anciens officiers du commerce, puis à des officiers mariniers, en particulier aux pilotes et ont recruté également des nouveaux officiers. Il reste cependant quelques anciens du Grand Corps. Un grand nombre de ces derniers furent écartés en l'an II et rayés des listes comme nobles. En 1796 et 1797, Truguet, luimême ancien du Grand Corps, ministre de la marine réintègre nombre de ces officiers : "Les états-majors sont devenus trop nombreux pour cette flotte réduite, et l'occasion est bonne pour se débarrasser des incapables. Truguet s'y emploie si bien qu'il peut faire rentrer dans les cadres actifs des officiers que la méfiance des Jacobins en avait écartés. Il y admet en même temps des jeunes gens capables de satisfaire à un examen d'entrée, peu rigoureux d'ailleurs - il n'y aurait pas de candidats - mais d'un niveau qui permette l'acquisition ultérieure des connaissances nécessaires. Et c'est le retour au recrutement des officiers de l'ancienne marine, dont la plupart venait des classes moyennes, bourgeoisie ou petite noblesse. Quand Truguet quitte le ministère en juillet 1797, après dix-huit mois de fonction, la réaction est complète. " *

Il convient cependant d'être prudent et ne pas exagérer l'épuration qu'aurait menée Truguet. Nous ne prendrons que l'exemple de deux officiers, l'un ignoré, l'autre illustre. Jean-François Achard, après avoir navigué au commerce de 1775 à 1781, entre dans la marine royale comme matelot en 1782. Aide-pilote en 1783, timonier en 1792, il est nommé lieutenant de vaisseau en ventôse an II. En juillet 1793, à Toulon il a « déserté du brick le Hazar pour joindre l'armée républicaine aux ordres du général Carteaux où j'ai pris du service avec l'agrément du ministre de la marine et j'ai coopéré à la réduction des rebelles marseillais et à notre entrée dans cette ville après avoir rallié les marins et m'être mis à leur tête pour réduire les infâmes qui avaient livré Toulon, commandant au siège de cette place la redoute des hommes sans peur et la compagnie des canonniers jacobins " ". Malgré une mauvaise appréciation ", Achard est conservé dans la marine du Directoire. Ce n'est que sous le Consulat qu'il sera écarté. En l'an XI, Jean-François Achard, arrêté par la police, se présente comme lieutenant de vaisseau. À une demande de renseignements, le ministère répond que cet officier "signalé par ses chefs comme un officier turbulent et insubordonné, a été réformé à l'organisation de l'an IX ». Une note de la main du ministre, qui a pu connaitre Achard lors de l'expédition d'Égypte, apporte un éclairage plus précis : « Achard a toujours eu la renommée d'un partisan reconnu du système de 1793. " "

Infernet, un des héros de Trafalgar, fut, malgré sa réputation, conservé par le Directoire. Lors de la réorganisation de l'an IX, Masséna intervient pour obtenir sa mise en activité. Le ministre, Forfait, retrace pour Bonaparte la carrière de cet officier : " Âgé de quarante-deux ans, matelot en 1776, lieutenant de frégate auxiliaire en 1781, lieutenant de vaisseau en 1793, capitaine de frégate le 16 floréal an II par les représentants du peuple alors en mission à Toulon. Je ne dois pas laisser ignorer, citoyen Premier Consul, que lorsqu'il fut question de l'organisation de l'an IV les notes envoyées alors des ports n'étaient point à l'avantage de cet officier. Elles portaient : la voix publique l'accuse de ne devoir son avancement qu'à l'influence des clubs, n'ayant point de conduite, des talents fort ordinaires et aimant le vin. Conservé cependant dans l'organisation de l'an IV. " "

24 Ces deux exemples illustrent le fait que les «officiers du maximum " ne furent pas rayés systématiquement des listes sous le Directoire. De la même façon, le gouvernement ne réintègre aucun officier émigré ". Ce n'est que sous le Consulat, avec 
Forfait et surtout Decrès, qu'une telle politique d'exclusion des anciens officiers et de rappel des émigrés se mettra en place. Le régime directorial est plus indulgent pour ses adversaires si l'on en croit un certain Guette qui, en l'an IX, demande sa réintégration : " À l'époque du 18 fructidor de l'an V, plusieurs officiers militaires et civils de la marine employés au Havre ont été suspendus de leurs fonctions sur des accusations vagues et calomnieuses. Presque tous ont obtenu leur réintégration $"^{\text {s" }}$.

Manquant d'argent, de matériel, d'hommes, la marine est impuissante à rivaliser avec les Anglais. Le Directoire se contente de bonnes paroles comme celles de Sieyès de passage à Anvers : "Il nous a assuré qu'il concourrait de tous ses moyens à mettre la marine française sur un pied formidable dans ces parages. ${ }^{\text {s" }}$

Lorsqu'en nivôse an VII, une frégate anglaise s'échoue sur la Chaussée des Saints, en face de Brest, un lougre et une corvette viennent au secours de l'équipage sans que les bâtiments français interviennent, en raison du calme selon Morard de Galle. On a d'ailleurs l'impression que la Royal Navy est maîtresse des eaux les plus proches des ports. "Presque tous les jours, deux ou trois frégates et une corvette ennemies paraissent à vue de l'île d'Ouessant et pénètrent quelquefois dans l'Yroise " ". Plus au sud, les Anglais viennent croiser tout aussi impunément : « Deux frégates et un vaisseau rasé ennemi se tiennent constamment dans le pertuis d'Antioche, plusieurs vaisseaux ennemis paraissant avoir établi leur croisière sur l'lsle Dieu. " "

En Méditerranée la situation n'est pas meilleure. La Corse paraît coupée du continent. Le chef des mouvements de Bastia dans une lettre datée du 12 pluviôse an VII signale que la dernière dépêche qu'il a reçue est du 25 brumaire. Le prestige de la marine est au plus bas, incapable de défendre les convois côtiers. À Cette, le commissaire du Directoire, exaspéré par l'inaction de la marine incapable de chasser un corsaire turc qui s'en prend aux convois, dénonce « le peu de zèle, l'insouciance et l'impéritie de la plupart des officiers qui commandent nos armements... jeunes gens sans expérience... Pourquoi ne pas employer des marins qui ont nombre de campagnes sur leur corps qui gémissent et souffrent de l'inactivité à laquelle on affecte de les condamner. Les bâtiments de la République se laissent jouer par des corsaires alors que sous l'Ancien Régime la seule annonce de l'envoi d'un bâtiment de l'État les faisait fuir. "s

Le ministre Bourdon de Vatry est tout aussi pessimiste que son prédécesseur et il avoue même qu'une victoire bien improbable sonnerait le glas de la marine. "Une action quelconque désemparerait ce qui reste de bâtiments, elle nous priverait d'un grand nombre de marins, nos magasins dépourvus ne pourraient remplacer les approvisionnements et les munitions que le feu de l'ennemi détruirait, de manière même qu'une victoire nous serait funeste et qu'elle paralyserait presque indéfiniment nos forces navales. $"$ "

Une marine conformiste?

Les relations officielles révèlent le loyalisme ou la prudence des marins, par exemple après le 18 fructidor : «Il n'est personne ici parmi les individus attachés au service de la marine qui n'ait témoigné sa satisfaction... si dans le nombre quelques-uns regrettent encore l'Ancien Régime, conservant dans leur âme l'espoir de le voir renaitre et se sont associés aux contre-révolutionnaires qui ont conçu le projet insensé de le faire revivre, aucun d'eux n'a manifesté son opinion au dehors " ". On comprend ces précautions lorsque le commandant d'armes comme à Lorient est un républicain, D'Albarrade, qui avoue : «Les royalistes ne se font pas connaître de moi. »" 

les escadres, on peut comparer les cérémonies où furent lues les proclamations des consuls en brumaire, celles d'acceptation de la Constitution en frimaire avec les fêtes et cérémonies qui ont scandé la vie des ports en 1799.

31

Au Havre par exemple, après les fêtes du 2pluviôse et du 26 messidor, on commémore le 9 Thermidor puis le 1er vendémiaire, on célèbre l'anniversaire de la République avant d'organiser le 11 du même mois une pompe funèbre en l'honneur du général Joubert et de saluer par des salves d'artillerie quatorze jours plus tard les victoires des armées de la République et le retour en France du général Bonaparte " ". Le déroulement de ces cérémonies n'est pas laissé au hasard si l'on en croit Nielly, commandant des armes à Lorient qui souligne qu'» autant que possible, nous avons exécuté le programme du ministre de l'Intérieur dont vous nous avez prescrit de suivre les dispositions " ". À Rochefort, les travaux de l'arsenal continuent le 26 messidor et l'ordonnateur s'en explique auprès du ministre et rend responsable la municipalité qui, ayant mal interprété les consignes du ministre de l'Intérieur, pensait qu'il fallait regrouper les cérémonies du 14Juillet, 10 Août, 9 Thermidor et 18 fructidor dans une fête unique le 10thermidor.

À ces fêtes la marine participe aux côtés des autorités civiles et de la population. À Brest, le 2 pluviôse, la cérémonie débute dans le temple des fêtes nationales en présence des autorités civiles et militaires, dans le temple de la commune à Rochefort. Le 26 messidor, au Havre, le cortège est formé par les marins mais aussi par la cavalerie, la garde nationale et des groupes de citoyens portant des étendards ". Le même jour le discours est prononcé par le président de l'administration municipale. La marine prête un concours matériel faisant construire par les charpentiers de marine un autel de la patrie ou en érigeant un cénotaphe, "dont la forme et la décoration rappelaient à tous les spectateurs le but qui les rassemblaient et le sentiment de vengeance dont ils devaient se pénétrer " ("), en l'honneur de Bonnier et Roberjot ". Il en est de même à Brest où " rien n'a été épargné par la marine qui pouvait contribuer à rendre cette fête funèbre aussi touchante que possible. Elle en a fait tous les frais " ". Le cénotaphe de marbre noir porte des inscriptions vengeresses: Guerre à mort à l'infâme Maison d'Autriche. Leurs mânes seront vengés ". Le faste des cérémonies dépend nécessairement de l'importance du port et, dans sa relation, le commandant des armes de Bordeaux souligne que la fête a eu lieu "avec toute la dignité possible et autant qu'un port secondaire peut le permettre" ". Cet officier semble peu disposé à organiser des festivités grandioses si l'on en croit une plainte des administrateurs de la ville auprès du ministre de l'Intérieur. Selon les Bordelais le commandant veut, par économie, interdire les salves d'artillerie ${ }^{20}$.

Ce sentiment d'union est renforcé par l'organisation d'un banquet aussi bien au Havre qu'à Anvers où, le 2 pluviôse an VII, 116 personnes, administrateurs et militaires se réunissent et où chacun participe en proportion de ses appointements donnant l'image "d'une grande famille très unie " ". À Flessingue les autorités municipales sont également invitées à partager ce « repas fraternel " .

Un autre moment fort de la cérémonie est la plantation d'un arbre de la liberté en particulier lors des fêtes célébrant la «juste punition du dernier roi des Français ». L'érection de cette "tige emblématique ", comme l'écrit le contre-amiral Lacrosse, 
présente une forte portée symbolique. À Ostende, cette plantation ne peut se faire devant le local de l'administration qui n'est pas propriétaire des lieux et qui juge l'endroit «peu convenable ». Cette plantation a donc lieu au quai des pêcheurs, « là où jadis s'élevait un mât surmonté d'un aigle impérial et là nous avons placé au lieu même où existait ce mât un jeune chêne décoré de rubans tricolores... Substitution à un signe d'esclavage celui de la liberté " ". À Bordeaux, l'arbre de la liberté ne peut que stimuler le zèle du personnel de la marine, "arbre sacré qui doit couvrir de son ombre les ouvriers de l'arsenal et lui donner cette activité si nécessaire aux opérations maritimes. " $"$

L'ensemble se termine le plus souvent par l'inévitable serment de haine à la royauté, à l'anarchie, d'attachement inviolable à la République et à la Constitution de l'an III, « la main droite levée et l'épée à la main " "s. À Nantes, les autorités maritimes participent, avec les différents membres du département à la plantation d'un arbre de la liberté place Buffon, « ensuite, toutes les personnes attachées à la marine se sont séparées des autorités constituées pour aller planter un autre arbre dans l'intérieur du port conformément aux ordres du ministre. ${ }^{20}$

Dans les cortèges et les rassemblements, les groupes sont souvent porteurs de bannières et d'étendards. Ceux-ci peuvent être des signes de reconnaissance comme à Brest avec les députations des ateliers et des vaisseaux, "les premiers portent l'emblème de leurs travaux, les autres un pavillon national " ". Au Havre, lors de la célébration du 14 Juillet, on peut lire : «Liberté conquise le 14 Juillet 1789; Gloire à la République; La République triomphe de tous les tyrans; Au 14 Juillet impatient d'une tyrannie qui avait pesé sur quatorze siècles le peuple français se lève et invoque une constitution " ". Aux panneaux et bannières se mêlent des acclamations qui s'en prennent à l'ennemi, c'est-à-dire, pour les marins, à l'Anglais : "Vive la République, Périssent ses ennemis, Périsse le gouvernement anglais. " "

La plus belle allégorie demeure celle qui figure sur le transparent qui décore la maison de la marine d'Anvers au 2 pluviôse. "Deux monstres se ressemblant parfaitement étaient à moitié écrasés et liés l'un à l'autre sous un énorme rocher. Le premier, image du royalisme, laissait tomber un masque qui cachait ses traits hideux. Il portait d'une main un bouquet de fleurs du milieu desquelles on voyait sortir la tête d'un serpent. De l'autre main il présentait des billets sur lesquels on lisait Banque: Bill. L'autre monstre était sans masque, allait plus droit au but, l'anarchie tient un poignard et une torche. Tous deux sont renversés sur des écussons, des sceptres, des armoiries, des missels, des couronnes, des trônes et des mitres, le tout étant cassé et brisé. Le rocher sert de piédestal au Temple de la Victoire qui porte autant de colonnes que la République a rendu de peuples à la liberté. La principale colonne est dédiée à la République, chacune des autres colonnes est dédiée à une république amie, la dernière étant la république piémontaise " ". Ces thèmes des deux périls, véritable lieu commun, se retrouvent, jusque dans les attributs de l'anarchie, dans le rapport du commandant d'armes de Brest après le 18 fructidor. «On voyait d'un côté le royalisme lever sa tête audacieuse et travailler ouvertement à frapper les fondements de la République pour ériger un trône sur ses débris, de l'autre le fanatisme aiguisant de nouveau les poignards, cherchait à semer parmi nous des divisions intestines et à y exciter les brandons de la guerre civile. " 

et des jeux de brigue, mâts graissés de suif, au milieu d'un grand concours populaire. "Tous les quais étaient complètement garnis par une multitude de citoyens qui pour s'y procurer des places s'y étaient rendus de bonne heure, les fenêtres des maisons ayant une vue sur le bassin étaient également garnies " ". L'allégresse est d'autant plus de mise sur les bâtiments lorsque les autorités font distribuer double ration d'eau de vie pour le déjeuner et double ration de vin pour le dîner, comme le fait Renaudin, commandant des forces navales de Brest pour l'anniversaire du 21 janvier. On peut voir également un signe de cette union dans la disposition même des cortèges. À Rochefort, les autorités prennent place au centre d'une colonne disposée par peloton de huit hommes de front sur trois rangs dont le premier est composé des troupes d'artillerie de marine, le deuxième de la garde nationale et le troisième de la 3e demi-brigade d'infanterie légère et ainsi de suite. De plus, « les drapeaux des différents corps étaient unis. »"

L'union est cependant loin d'être l'unanimité si l'on en croit les précautions prises par les autorités d'Ostende qui choisissent un lieu situé près d'un corps de garde pour $\mathrm{y}$ planter l'arbre de la liberté ou l'acte de malveillance qui détruit, la veille du 26 messidor, l'autel de la patrie construit par les charpentiers de marine au Havre.

Enfin si la lutte contre les deux périls est soulignée et imagée, à la lecture des comptes rendus et rapports, le royalisme semble être l'ennemi principal. En effet, la commémoration du 9 Thermidor ne fait l'objet que de remarques incidentes et ne paraît pas avoir été l'occasion de pompes importantes. À Lorient, seule allusion que nous rencontrons, il s'agit d'un repas civique qui, débutant à six heures du soir, dure une heure et demie. Nielly, commandant des armes dans ce port, se défend contre des attaques dont le sens nous demeure inconnu : «Il se peut que la malveillance se plaise à le présenter sous un aspect défavorable " "et, pour se justifier, envoie au ministre la liste des toasts qui ont été portés : «À la République, À la Constitution, À la ruine de la royauté et de l'anarchie, À la chute de Robespierre, À la paix, Aux alliés, À l'union de tous les Français ».

Par-delà la satisfaction officielle, l'approbation de rigueur, la correspondance laisse deviner, face au manque d'hommes et d'argent, une certaine exaspération. Selon l'amiral Bruix, le commandant des armes de Toulon, le contre-amiral Vence ne manifeste pas un très grand respect pour les autorités : « Il s'est permis de fronder en présence de plusieurs officiers de l'armée le plan de campagne qu'il suppose avoir été arrêté par le Directoire exécutif. »"

par le découragement comme l'ordonnateur du port de Brest qui se sent «abandonné à (lui) même sans secours et sans espoir d'en obtenir, je semble soutenir le poids énorme qui m'accable. »"

43 Le commandant d'armes de Rochefort, l'amiral Martin, dans une lettre au ministre s'en prend aux réformateurs qui ne connaissent pas la question et sa référence aux hommes de loi semble un écho à ceux qui s'en prenaient au gouvernement d'avocats : «L'on crie contre la marine à cause des revers qu'elle a éprouvés depuis le commencement de la guerre de la Liberté. On imagine qu'on fait un matelot comme un soldat et tous les fameux qui ont parlé sur cette partie de la force publique n'ont pas dit un seul mot en faveur des marins. La vérité n'est pas connue des hommes de loi qui jusqu'à présent ont été chargés de la désorganiser plutôt que de la conserver au gouvernement. Les 
hommes qui les connaissent qui ont partagé leurs peines n'ont jamais été consultés. Mais que de choses à dire sur toutes les organisations acceptées ou projetées... " ${ }^{87}$

La subordination ne semble pas être de rigueur si l'on en croit la correspondance ministérielle. En pluviôse an VII, Bourdon écrit à l'ordonnateur de Brest pour lui annoncer que « le munitionnaire général de la marine réalise en ce moment à Brest un million d'écus et un autre million et demi qui doit se grossir de tous les fonds qui se trouvent dans les caisses depuis Alençon jusqu'à Brest est maintenant en route et sous bonne escorte " ${ }^{s}$. Le ministre recommande à l'ordonnateur de régler les soldes en numéraire et les autres dettes avec des traites. En effet, le ministre avait toujours refusé les offres de traites des commissaires de la Trésorerie mais l'ordonnateur, sans consulter son ministre, avait obtenu de ces commissaires des traites. Bourdon, dans sa lettre, évoque même des remontrances que l'ordonnateur lui aurait faites «contre toutes les règles de la subordination... je ne vous dissimule pas que votre conduite en cette occasion est très répréhensible. " "

Une marine satisfaite par le coup d'État?

La participation la plus spectaculaire de la marine au 18 Brumaire est le cheval, animal de Neptune, prêté par l'amiral Bruix à Bonaparte, « un cheval d'Espagne noir et d'une beauté remarquable mais ardent et rétif " ". Les conséquences du coup de force de Saint-Cloud se font sentir en deux temps dans les ports et les escadres. Fin brumaire les proclamations des Consuls sont suivies de serments alors que l'acceptation de la Constitution, fin frimaire, est l'occasion de cérémonies. Remarquons que les nouvelles autorités, en l'occurrence les commissions législatives, suppriment rapidement, avant même la mise en place des nouvelles institutions, les fêtes du 21 janvier, 18 fructidor, 9 Thermidor pour ne conserver que celles du 14 Juillet et du 1er vendémiaire.

Les ports les plus favorisés sont prévenus dès le 22 brumaire. Une dépêche arrivée à Port-Malo par télégraphe annonce que : «Le Corps législatif a nommé consuls pour remplacer le Directoire exécutif les citoyens Sieyés, Roger Ducos et le général Bonaparte. Il a nommé deux commissions de vingt-cinq membres pour chaque conseil et s'est ajourné jusqu'au 1er ventôse. Tout est tranquille et contenu à Paris. Les effets publics ont gagné prodigieusement " ". Le changement de gouvernement est porté à la connaissance des marins par l'affichage comme le montre l'exemple du Havre : « Nous avons reçu avec votre lettre du 21 de ce mois une proclamation des Consuls de la République. Suivant vos instructions nous nous sommes empressés d'en afficher des exemplaires à toutes les portes des bassins " ". L'annonce même des événements est l'occasion de rompre la belle unité affichée quelques mois plus tôt. Lorsque l'agent maritime d'Anvers écrit au ministre pour lui signaler l'arrivée d'un courrier extraordinaire apportant le décret rendu par le Conseil des Anciens en vertu des articles 102, 103 et 104 de la Constitution, il ajoute qu'il ne connait cette information que par l'affichage et manifeste une certaine aigreur d'avoir été tenu à l'écart: «La marine est aussi une force armée et les égards que les autorités constituées se doivent entre elles ne me paraissent pas avoir été observés en cette occasion. " "

La première caractéristique des cérémonies qui suivent le coup d'État est leur caractère, le plus souvent, proprement militaire. À Anvers, le 27 brumaire devant la maison de la marine illuminée, pavoisée et portant un transparent «La marine aux sauveurs de la patrie » sont lues les proclamations de Bonaparte du 19 et du 21 devant les troupes sous les armes et le nouveau serment est prêté par les officiers civils et militaires réunis aux troupes de terre ". Il en va de même au Havre ou, dans la même 
circonstance, se rassemblent les officiers d'administration, militaires, marins, ouvriers et troupes d'artillerie devant le bassin neuf ". À Dunkerque, les autorités civiles et la population sont tout aussi absentes, car devant l'arsenal seuls les troupes, les ouvriers et les gardiens forment un "bataillon quarré » ". À Nantes, la lecture de la proclamation et le serment du 1er frimaire ont lieu devant l'arbre de la liberté, mais celui de la marine, et l'armée est également présente : «J'ai invité le général, commandant la force armée, à mettre à ma disposition un fort détachement de troupes réglées avec de la musique pour accompagner les autorités réunies de la marine jusqu'à l'arbre de la Liberté qui est vis-à-vis du magasin général " ". Le commissaire de marine de SaintMalo a décidé de suspendre les travaux le 3nivôse, jour fixé pour l'acceptation de la Constitution " pour donner à cette cérémonie toute la solennité qu'elle méritait " ". Les équipages, "au son des airs qui conduisent les Français à la victoire" ", se rendent à l'arbre de la Liberté. Après avoir entendu la lecture des « lois » des 19 et 20 brumaire, «lois qui ont sauvé la patrie et qui l'ont retenue du gouffre dans lequel on voulait la précipiter ", ils prêtent serment, puis ce serment est à nouveau prononcé après le retour sur les bâtiments.

À Brest, la cérémonie d'acceptation de la Constitution, si elle est plus impressionnante par le nombre de participants, exclut également les autorités locales : « Aujourd'hui les officiers civils et militaires, les troupes, les marins et les ouvriers de l'arsenal ont au nombre de 30000 prêté d'un concert unanime le serment solennel de fidélité et de dévouement à la défense de la patrie " ${ }^{10}$. Le seul cas que nous ayons rencontré de participation des autorités civiles à la prestation de serment est celui de Lorient mais la formulation traduit bien le poids de la marine dans ce port : « Nous avons invité à cette cérémonie l'état-major de la place et l'administration municipale, tous les assistants ont répété le serment avec nous et tout s'est passé dans le plus grand ordre " "10. À Rochefort, la relation du commandant des armes ne mentionne pas les autorités civiles mais signale la présence massive d'habitants du port: «Une quantité innombrable de citoyens de cette commune qui avaient assisté à cette cérémonie y ont également contribué en se mêlant parmi les troupes et les ouvriers et partageant leurs vœux pour le maintien d'une Constitution qui, en terminant la Révolution, consolidera la gloire et la puissance de la République française " " ${ }^{\text {"xa }}$. Remarquons que Lorient et Rochefort sont deux ports où le poids de la marine est considérable.

Les discours reprennent des thèmes semblables: exaltation de l'unité face à la désunion, nécessité de l'ordre face au désordre, appel à un gouvernement fort qui conclura une paix glorieuse: "Substituer l'ordre au désordre, l'économie aux dilapidations, un gouvernement régulier à une constitution méconnue et violée par tous les partis, rapporter ces lois révolutionnaires, fléau des familles, cause de leur ruine, source de nos dissensions intérieures " ${ }^{100}[. .$.$] Le pacte social qui vous est présenté$ doit en centralisant la force et l'action de la puissance publique faire succéder incessamment l'ordre au chaos, le calme aux agitations, la paix aux funestes fléaux de la guerre, alors les Français occupant le premier rang des peuples de l'Europe jouiront d'une félicité, encore quelques instants et nous en goûterons les délices que notre imagination ne peut même nous décrire qu'imparfaitement. " "10

Les références propres à la marine sont rares. Seul l'agent maritime d'Anvers y fait allusion en insistant sur la sécurité et la stabilité qu'apporte le nouveau gouvernement. Il affirme également que les marins ne dépendront pas de civils, allusion sans doute à un projet de Pléville Le Pelley lorsque celui-ci était ministre de la marine, projet qui ne 
fut pas mis en application : «Marins, vous ne verrez plus les vaisseaux de la République, instruments nécessaires à votre gloire passer dans les mains d'avides armateurs et transformés en corsaires [...] officiers de tous grades vous ne craindrez plus les réformes en masse, les destitutions arbitraires. " $"$ "105 Le changement de régime est aussi l'occasion de discours en d'autres lieux que les grands ports. Dans le Sud-Ouest, un sous-commissaire de marine de Bordeaux tient aux marins du quartier maritime de Cazères un discours où se mêlent la défense de la République, les intérêts des marins et l'exaltation du héros : « La Constitution de l'an III par ses vices n'avait pu conserver vos droits et assurer le bonheur général, bientôt même, elle eût fait place à un tyran couronné qui, dans le sang et sur les cadavres des républicains, eût assis son trône [...] Sauveurs de la République, espérons que les promesses qui, tant de fois, vous ont été faites de vous solder de vos campagnes seront tenues [...] L'un d'eux, le vainqueur d'Arcole, de Lody et de l'Égypte a connu et partagé vos souffrances... il vous rendra justice. " "

52 Si dans les cérémonies de l'an VII que nous avons évoquées, le thème de l'union était très présent, les proclamations et serments qui suivent le 18 Brumaire renvoient à l'unanimité. Au Havre l'acceptation de la nouvelle Constitution se fait par écrit et en groupe: "Le cortège était de retour à la maison commune où les registres d'acceptation et de non-acceptation étaient ouverts, ceux qui le composaient ont de suite émis leur vote pour l'admission du pacte social qui en terminant la révolution doit assurer la félicité ». Un de ces registres a dû rester vierge si l'on en croit le procèsverbal, envoyé quelques jours plus tard au ministre, et qui annonce «l'acceptation votée à l'unanimité par tous les administrateurs et militaires, soldats de marine et marins employés à ce port " "'. Il semble que l'acceptation se fasse en deux temps. La lecture des relations donne à penser que l'approbation a lieu par la signature de registres, mais seuls les officiers et les administrateurs paraissent remplir cette formalité alors que la Constitution est considérée comme acceptée par les marins par acclamations. Ainsi à Bordeaux « quoique le vœu de la loi du 23 frimaire se trouve ainsi rempli par notre acceptation consignée sur les registres ouverts par la municipalité » $\left.{ }^{(*)}\right)$, les autorités maritimes suivent les instructions du ministre, réunissent le personnel de la marine et lisent la nouvelle Constitution, lecture « acclamée par l'universalité des citoyens " ${ }^{\prime \prime}$. Cependant le déroulement est parfois confus comme à Port-Malo où le chef des mouvements veut faire placer des registres d'acceptation et de nonacceptation sur chaque bâtiment ".

53 Ce concert unanime que nous avions signalé pour les 30000 hommes de Brest se retrouve sur le plus modeste bâtiment. Par exemple, à Ostende, l'enseigne de vaisseau Jacques Villeneuve qui commande une canonnière rassemble l'équipage à l'arrière du bateau. Les officiers mariniers lui « ont tous déclaré accepter la nouvelle Constitution de la République française ». Le procès-verbal est suivi par les signatures des membres de l'équipage, signatures parfois remplacées par une croix à côté de laquelle on a ajouté " marque ordinaire de signature " "'. Il n'apparait pas toujours nécessaire de recourir au registre et au procès-verbal paraphé. À Dunkerque, aussi bien en brumaire qu'en frimaire, le personnel de la marine forme un carré, le commissaire de la marine fait prêter serment au cours d'une première cérémonie, un mois plus tard, les hommes étant dans la même disposition, après la lecture de la nouvelle Constitution, un accord oral et unanime suffit: "Tous, d'une voix unanime, ont, par acclamation, Vive la République, Vive la Constitution, accepté ce nouveau pacte social qui voit consolider et 
affermir la République " ". Il en va de même à Saint-Malo, Brest et Nantes où la Constitution est adoptée à l'unanimité par acclamation. Dans ces ports, nous n'avons trouvé aucune allusion à des registres. Il convient de demeurer prudent face à un si bel enthousiasme. Quelques semaines après le coup d'État, le contre-amiral Perrée signale que les marins « ont été tant de fois trompés qu'ils sont devenus sourds à la voix de la Patrie. $"{ }^{13}$

Le dernier élément marquant nous semble être une personnalisation accrue. Sous le Directoire, les gouvernants étaient rarement cités et de façon anonyme et collective comme dans le toast porté le 2 pluviôse an VII, au Havre, au Directoire et aux deux Conseils. Après le 18 brumaire, le mouvement paraît irréversible. Si le contre-amiral Delmotte reste très général en voyant «à la tête du gouvernement le patriotisme et le talent qui fondent notre espoir pour l'avenir" "14, d'autres sont plus précis en rapportant les « acclamations les plus affectueuses concernant le général Bonaparte » "15. L'agent maritime d'Anvers est plus lyrique encore lorsqu'il évoque « ces hommes déjà supérieurs à leur siècle qui, touchés des maux de leur patrie, ont projeté à jamais de la délivrer des factions et des factieux ». L'auditoire devait reconnaître ceux qui n'étaient pas nommés : "Cet ami de la liberté qui, dès l'aurore de la Révolution fit connaître au peuple ce qu'il était et ce qu'il devait être et prend les rênes du gouvernement dans un moment très difficile après avoir assuré par la sagesse de ses négociations la neutralité d'un des plus puissants princes de l'Europe le vainqueur de l'Italie et de l'Égypte, le jeune héros dont toute la carrière des armes est une série de prodiges et dont la conduite politique est sans tache. " ${ }^{116}$

L'étude des cérémonies qui ont précédé et suivi le 18 Brumaire, une des seules expressions politiques dont nous disposions, révèle donc une militarisation croissante, une affirmation de l'unanimité et une personnalisation du gouvernement. Ce sont là des tendances qui ne feront que s'accentuer. Le dénuement de la marine et des marins était tel dans les derniers mois du Directoire que l'on peut comprendre le discrédit dans lequel il était tombé et l'espoir qu'a pu soulever un changement de régime. De ce renouveau témoigne le rapport envoyé par l'ordonnateur de Brest, pour présenter l'état de ce port, et qui commence par ces mots: "Au moment où le gouvernement investi de la confiance générale prépare une réorganisation nécessaire de toutes les parties du corps social et politique, il est essentiellement du devoir de l'homme en place de faire connaître la situation dans laquelle se trouve l'administration dont il est chargé. " ${ }^{117}$

Il semble donc que la marine ait accueilli assez favorablement, pour autant que l'on puisse juger, le coup d'État du 18 Brumaire. Cette approbation peut s'expliquer par le conformisme politique du corps mais surtout par l'état de déliquescence dans lequel le Directoire avait laissé la marine. Des marins mal équipés, mal armés, mal payés ne pouvaient qu'accorder un préjugé favorable à un nouveau régime et à son programme d'ordre et de grandeur. Ces promesses de Brumaire furent-elles tenues par le Consulat et l'Empire ? C'est là un autre problème. 


\section{NOTES}

1.A.N., AF III 205.

2.A.N., AF III 205, lettre du 10 thermidor an V.

3.A.N., AF III 205, rapport du 22 messidor an V.

4.Ibid.

5.A.N., AF III 205, rapport du 15 ventôse an VI.

6.A.N., AF III 205.

7.A.N., BB3/153, lettre du 15 nivôse an VII.

8.Ibid., lettre du 19 frimaire an VI.

9.Ibid., lettre du 23 frimaire an VIII.

10.Ibid., lettres des 29 frimaire an VII et 21 brumaire an VIII.

11.A.N., BB3/154, lettre du 13 brumaire an VIII.

12.A.N., BB3 $/ 155$, lettre du 30 thermidor an VII.

13.A.N., BB3/156, lettre au ministre, 19 thermidor an VII.

14.A.N., BB4/130, lettre du 16 prairial an VII.

15.A.N., BB3/157, lettre du 5 thermidor an VII.

16.A.N., BB4/137, lettre du chef de division Sibille, commandant des forces navales de l'armée d'Italie, 26 fructidor an VII.

17.A.N., BB4/131, lettre au président du Directoire exécutif, 4 germinal an VII.

18.A.N., BB4/131, lettres des 28 et 30 floréal.

19.A.N., BB3/153, lettre du 29 ventôse an VII.

20.Ibid., lettre du 4 fructidor an VII.

21.A.N., BB3/156, lettre du 24 vendémiaire an VIII.

22.A.N., BB3/158, lettre de l'ordonnateur, 7 thermidor an VII.

23.A.N., AF III 205, lettre de l'ordonnateur de Brest, 3 thermidor an VII.

24.A.N., BB4/132, lettre du contre-amiral Linois, chef d'état-major de l'amiral Bruix au ministre, 7 nivôse an VIII. Des remarques sibyllines du contre-amiral Pérée, qui vient de prendre à Toulon le commandement d'une division chargée de ravitailler Malte, laissent à penser que l'administration n'était pas au-dessus de tout soupçon : «Je me tairai avec peine sur les travaux de l'arsenal, ce qu'on y fait est bien loin de balancer ce qu'on y dépense et le nom d'arsenal la fait encore augmenter. Je crois en dire assez. » BB4/136, lettre du 26 frimaire an VIII.

25.A.N., BB3/156, lettre du commandant des armes, 25 vendémiaire an VIII.

26.A.N., BB3/155, lettre du 24 thermidor an VII.

27.A.N., AF III 205, lettre de l'ordonnateur de Brest, 3 thermidor an V.

28.A.N., AF III 205, rapport du ministre, 1er ventôse an V.

29.Ibid., lettre du 28 thermidor an VII.

30.A.N., BB3/157, lettre du 19 nivôse an VII.

31.A.N., BB4/134, lettre du commandant des armes et de l'ordonnateur de Rochefort au ministère, 11 germinal an VII.

32.A.N., BB4/131, lettre au président du Directoire exécutif, 4 germinal an VII.

33.A.N., BB3/153, lettre de Morard de Galle, 19 nivôse an VII.

34.A.N., BB3/158, État de germinal an VII.

35.A.N., BB3/154, lettre du commandant des armes au ministre, 1er prairial an VII.

36.A.N., BB3/162, lettre du 2 fructidor an VII. 
37.A.N., BB3/153, lettre du 17 pluviôse an VII.

38.Ibid., lettre du 11 ventôse an VII.

39.A.N., BB3/157, lettre du 19 nivôse an VII.

40.A.N. BB4/138, lettre du commandant des armes de Toulon au ministre, 22 fructidor an VII.

41.A.N., BB3/159, lettre du 27 frimaire an VII.

42.Ibid., lettre du 11 brumaire an VIII.

43.A.N., BB3/155, lettre du 21 brumaire an VIII. Une lettre du ministre de l'Intérieur à son collègue de la Marine est éclairante sur la situation de ces départements et en l'occurrence du Morbihan. Le personnel de la Marine, comme les autres fonctionnaires, refuse de participer aux jurys, arguant de leur statut. « La formation du tableau des jurés deviendrait presque impossible, surtout dans ce département où la plus grande partie de ceux qui parlent et entendent le français occupent des fonctions publiques, dans un département dont un grand nombre d'habitants fanatisés ou égarés par le royalisme, ne peut exercer les importantes fonctions de juré avec cette impartialité que commande la justice et doit être par conséquent rejeté de cette liste. » (A.N., BB3/160, lettre du 15 vendémiaire an VIII).

44.A.N., BB3/156, lettre de l'amiral Martin, commandant des armes.

45.A.N., BB4/136, lettre du contre-amiral Perrée au ministre, 26 frimaire an VIII.

46.A.N., BB4/135, lettre au ministre, 26 prairial an VII.

47.Journal de bord du corsaire Plucket, p. 203.

48.A. THOMAzI, Napoléon et ses marins, p. 27.

49.S.H.M., série CC7, dossier Jean-François Achard.

50.« Les renseignements le font passer pour cabaleur, inquiet et porté à troubler et à bien des égards mauvais ", S.H.M., série CC7, dossier Jean-François Achard, note du 10 fructidor an IV.

51.A.N., CC1/184, lettre au ministre de l'Intérieur du 7 prairial an XI.

52.A.N., CC1/21, rapport du 15 messidor an IX.

53.N. BONNET-BERTRAND, Les officiers de Marine du Directoire, thèse, école des Chartes, 1984, pp.VII-6.

54.A.N., CC1/21, 16 ventôse an IX.

55.A.N., BB4/130, lettre de l'ordonnateur au ministre, 16 prairial an VII.

56.A.N., BB3/153, lettre de Morard de Galle, 15 vendémiaire an VIII.

57.A.N., BB3/156, lettre du commandant des armes de Rochefort, 25 prairial an VII.

58.A.N., BB3/162, lettre du 16 thermidor an VII.

59.A.N., BB4/131, rapport du ministre au Directoire, 29 thermidor an VII.

60.A.N., AF III 205, lettre du commandant d'armes de Brest au ministre, 5e jour complémentaire an $\mathrm{V}$.

61.A.N., AF III 205, lettre au ministre, vendémiaire an VI. « Aux yeux du gouvernement, et probablement avec raison, le péril royaliste est beaucoup plus dangereux dans la Marine que le péril jacobin... Les officiers de Marine, mis à part ces quelques cas (les proscrits du 18 fructidor dont Villaret Joyeuse), restent pour la plupart à l'écart des passions politiques : le relatif petit nombre de destitutions intervenues après le coup d'État du 18 fructidor, alors que le ministre Pléville de Pelley est décidé à une épuration sévère, en est la preuve, ainsi que la facilité avec laquelle la Marine accueille le 18 Brumaire et l'Empire. » (N.BONNET-BERTRAND, op. cit., pp. V, 28 et 32).

62.A.N., BB3/150, rapport du contre-amiral La Crosse, commandant d'armes. « Il y a donc, tout au long du Directoire, cinq grandes commémorations nationales : 14 Juillet, 
10 Août, 1er vendémiaire, 21 janvier, 9 Thermidor. Parfois s'y ajoutent ces commémorations passagères qu'impose l'événement, comme les fêtes funèbres de Hoche ou de Joubert, ou les fêtes triomphales comme le 20 nivôse an VI, celle de la paix continentale. » (M. Ozouf, La fête révolutionnaire, 1789-1799, Paris, 1976, p. 140).

63.A.N., BB3/154, lettre du 7 pluviôse an VII. « Ainsi se poursuit jusqu'à la fin de la décennie révolutionnaire un même rêve d'unanimité : que chaque citoyen reconnaisse partout le même plan, le même objet, les mêmes rites, les mêmes chants. » (M. ozouf, op. cit., p. 143).

64.A.N., BB3/149, compte rendu du chef de mouvement.

65.A.N., BB3/150, rapport du commissaire de Marine, 21 prairial an VII.

66. Le post scriptum de la lettre rendant compte de la cérémonie témoigne de l'immense détresse de la Marine : «Le cénotaphe a été construit à peu de frais et avec les vieux bois qui sont rentrés dans les magasins. "

67.A.N., BB3/153, lettre du commandant des armes Morard de Galle et de l'ordonnateur Najac au ministre.

68. Ce cénotaphe témoigne également de l'évolution artistique puisqu'il est formé de « deux cerceuils de granit égyptiens ».

69.A.N., BB3/157, lettre du 3 pluviôse an VII.

70.A.N., BB3/160, lettre du ministre de l'Intérieur, 10 fructidor an VII.

71.A.N., BB3/147, lettre de l'agent maritime au ministre, 3 pluviôse an VII.

72.A.N., BB3/163, lettre du commissaire du gouvernement.

73.A.N., BB3 $/ 148$, lettre de l'agent maritime au ministre.

74.A.N., BB3/157, lettre du commandant des armes et de l'ordonnateur, 3 pluviôse an VII.

75.A.N., BB3/163, lettre du commissaire du gouvernement.

76.A.N., BB3/155, lettre du chef des mouvements, 14 pluviôse an VII.

77.A.N., BB3/153, lettre du commandant des armes et de l'ordonnateur, 2 pluviôse an VII.

78.A.N., BB3/149, lettre du chef du mouvement au ministre.

79.Fête du 2 pluviôse à Brest.

80.A.N., BB3/147, lettre de l'agent maritime au ministre, 3 pluviôse an VII.

81.A.N., AF III 205, lettre au ministre du 5e jour complémentaire an V.

82.A.N., BB3/147, lettre du chef maritime au ministre.

83.A.N., BB3/156, lettre du 3 pluviôse.

84.A.N., BB3 $/ 154$, lettre du 10 thermidor an VII.

85.A.N., BB4/131, lettre du 30 floréal an VII.

86.A.N., BB4/134, lettre de Najac au ministre, 23 brumaire an VIII.

87.A.N., BB3/156, lettre du 25 vendémiaire an VIII. Les points de suspension sont de l'amiral Martin.

88.A.N., BB4/134, 16 pluviôse an VII.

89.Ibid.

90.A. VANDAL, L'avènement de Bonaparte, t. I, p. 246. Le rôle de l'ancien ministre de la Marine ne s'est pas limité à ce prêt. Dès le retour d'Égypte, il fait partie du groupe qui se rallie à Bonaparte avec Talleyrand, Roederer, Regnault de Saint-Jean-d'Angély, Maret, Boulay de la Meurthe et Réal. C'est à Bruix que Ouvrad, fournisseur de la Marine, s'adresse pour proposer ses services le 18 brumaire. Avec Talleyrand, l'amiral arrache, sans trop de peine, la démission de Barras.

91.A.N., BB4/130. 
92.A.N., BB3/149, lettre du commissaire principal au ministre, 28 brumaire an VIII.

93.A.N., BB3/142.

94.A.N., BB3/143, lettre de l'agent maritime au ministre, 29 brumaire an VIII.

95.A.N., BB3/149, lettre du commissaire principal au ministre, 28 brumaire an VIII.

96.A.N., BB3/148, lettre du commissaire principal au ministre, 29 brumaire an VIII.

97.A.N., BB3/155, lettre du chef des mouvements.

98.A.N., BB3/152, lettre du 5 nivôse an VIII.

99.A.N., BB4/130.

100.A.N., AF IV 1444, adresse de l'armée navale du 27 frimaire an VIII signée de Morard de Galle, commandant d'armes et de l'ordonnateur Najac.

101.A.N., BB3/154, lettre du commandant des armes, 5 frimaire an VIII.

102.A.N., BB3/156, lettre du commandant des armes, 5 nivôse an VIII.

103.Il s'agit sans doute d'une allusion à la loi du 24 messidor an VII, dite loi des otages.

Dans les départements déclarés en état de trouble par les Conseils, les autorités locales

pouvaient désigner des otages parmi les nobles, les parents d'émigrés ou de brigands notoires. Ces otages devaient se constituer prisonniers dans les dix jours. En cas d'assassinat ou d'enlèvement d'un fonctionnaire ou d'un défenseur de la patrie ou de ses parents proches, quatre otages seraient déportés. En outre ces otages étaient considérés comme pécuniairement responsables des dégâts causés par les brigands. Cette loi est rapportée dès le 22 brumaire.

104.A.N., BB3/143 et BB3/149, discours de l'agent maritime d'Anvers et du chef d'étatmajor du Havre.

105.A.N., BB3/143, discours de l'agent maritime d'Anvers, le 28 brumaire an VIII. Lettre au ministre du 29 brumaire. L'unanimité ne devait pas régner dans l'ensemble du corps. L'ordonnateur de Toulon se plaint souvent de l'» abus de pouvoir exercé par des ouvriers et des marins subalternes qui se trouvent revêtus de l'écharpe municipale quoique salariés par la République pour des fonctions dont ils ne s'occupent pas ». BB4/134, lettre du 26 prairial an VII.

106.A.N., BB3/157.

107.A.N., BB3/149, lettre du chef de mouvement au ministre et procès-verbal du 3 nivôse an VIII.

108.A.N., BB3/156, lettre du commandant des armes de Bordeaux.

109.Ibid.

110.A.N., BB4/130, lettre du chef des mouvements de Port-Malo au capitaine de vaisseau Willaumez, 3 nivôse an VIII. La loi du 23 frimaire prévoyait l'ouverture de registres dans chaque commune, au secrétariat de toutes les autorités, aux greffes de tous les tribunaux, entre les mains des agents communaux, des juges de paix et des notaires.

111.A.N., BB3/148, rapport de l'agent maritime au ministre et procès-verbal, 3 nivôse an VIII.

112.A.N., BB3/148, lettres du commissaire principal au ministre.

113.A.N., BB4/134, lettre du 26 frimaire an VIII. Les résultats officiels donnèrent trois millions de oui contre un peu moins de deux milliers de non. « Les oui en réalité n'ont pas dépassé 1600000. La falsification s'est opérée en gonflant systématiquement de 900000voix les résultats départementaux et en ajoutant, pour faire bonne mesure, autant de oui que de militaires, soit 500000, alors que ceux-ci n'ont pas été consultés. » (Claude LANGLOIS, « Napoléon Bonaparte plébiscité », in L'élection du chef de l'État en France de Hugues Capet à nos jours. Entretiens d'Auxerre, 1987). 
114.A.N., AF IV 1444, lettre du contre-amiral commandant l'armée navale de Brest au ministre, 15 frimaire an VIII.

115.A.N., BB3/148, lettre du commissaire principal de Dunkerque au ministre, 29 brumaire an VIII.

116.A.N., BB3/143, discours du 28 brumaire an VIII. Lettre au ministre du 29 brumaire. 117.A.N., BB4/134, rapport du 3 frimaire an VIII.

\section{RÉSUMÉS}

À la veille du 18 Brumaire, la marine paraît dans un état pitoyable. Les difficultés financières ne permettent pas d'armer des navires, marins et ouvriers ne touchent plus leurs soldes. Les levées d'hommes sont insuffisantes et la désertion sévit. Les ports de l'Ouest sont isolés en raison des troubles qui règnent dans l'arrière-pays. Cependant les cérémonies officielles témoignent d'un attachement au gouvernement ou, tout au moins, d'un conformisme certain. Pour autant qu'on puisse en juger, le coup d'État est assez bien accueilli. Mais les cérémonies qui suivent et les prestations de serment se caractérisent par une militarisation croissante et par une unanimité plus ou moins contrainte.

The French Navy and 18 Brumaire. On the eve of 18 Brumaire, the Navy seemed in a sorry state. Financial difficulties prevented the ships from being commissioned, while sailors and workmen went unpaid. Levies were inadequate and desertion was rife. The Western ports were isolated because of the unrest inland. Official ceremonies, however, demonstrated loyalty to the Government, or at least a readiness to conform. As far as can be judged, the Coup on the whole was well received. But the ceremonies which ensued and the oaths of allegiance proved increasingly militaristic and the unanimity was somewhat forced.

La marina francese alla data del 18 Brumaio. Alla vigilia del 18 Brumaio la marina pareva in uno stato pietoso. Le difficoltà finanziarie non permettono di aramre delle navi, marinai e operai non percepiscono più salario. Le leve di uomini sono insufficienti e dilaga la diserzione. I porti dell'Ovest sono isolati a causa dei disordini che regnano nel retroterra. Ciò nonostante le cerimonie ufficiali testimoniano un attaccamento al governo o, per lo meno, un sicuro conformismo. Per quanto se ne può giudicare, il colpo di Stata è piuttosto ben accolto. Ma le cerimonie che seguono e le prestazioni di giuramento sono caratterizzate da una militarizzazione crescente e da una unanimità più o meno coatta.

En vísperas del 18 brumaire, se encuentra la marina en estado lamentable. No le permitenarmar barcos sus dificultades hacendísticas, y no cobran sueldos los marineros y obreros. Son insuficientes los reclutamientos e impera la deserción. Quedan aislados los puertos del oeste por los disturbios de tierra adentro. Sin embargo, las ceremonias oficiales demuestran cierta fidelidad al gobierno o, por lo menos, un evidente conformismo. De pocler opinar sobre el caso, parece beneficiar de favorable acogida el golpe de Estado. Pero las ceremonias y juramentos que le siguen se caracterizan por una creciente militarización, y una unanimidad más o menos forzada.

Die französische Marine am 18. Brumaire. Kurz vor dem 18. Brumaire scheint die französische Kriegsmarine in einem jämmerlichen Zustand zu sein. Die Finanzschwierigkeiten erlauben nicht 
mehr, Schiffe auszurüsten, Matrosen und Arbeiter bekommen keinen Lohn mehr. Die Aushebungen sind unzureichend und die Desertionen zahlreich. Die atlantischen Häfen sind wegen der Unruhen, die im Hinterland herrschen, isoliert. Die offiziellen Zeremonien zeugen immerhin von Treue zu der Regierung, wenigstens von einer gewissen Anpassung. Soweit man es beurteilen kann, wird der Staatsstreich ziemlich positiv aufgenommen. Aber die darauf folgenden Zeremonien und die Vereidigungen zeigen eine wachsende Militarisierung und eine mehr oder weniger erzwungene Einstimmigkeit.

\section{AUTEUR}

\section{PIERRE LÉVÊQUE}

Université de Paris I 